\title{
LETTER
}

\section{Experimentally increased group diversity improves disease resistance in an ant species}

\author{
Anabelle Reber, Grégoire \\ Castella, Philippe Christe and \\ Michel Chapuisat* \\ Department of Ecology and \\ Evolution, Biophore, UNIL- \\ Sorge, University of Lausanne, \\ 1015 Lausanne, Switzerland \\ *Correspondence: E-mail: \\ michel.chapuisat@unil.ch
}

\begin{abstract}
A leading hypothesis linking parasites to social evolution is that more genetically diverse social groups better resist parasites. Moreover, group diversity can encompass factors other than genetic variation that may also influence disease resistance. Here, we tested whether group diversity improved disease resistance in an ant species with natural variation in colony queen number. We formed experimental groups of workers and challenged them with the fungal parasite Metarbizium anisopliae. Workers originating from monogynous colonies (headed by a single queen and with low genetic diversity) had higher survival than workers originating from polygynous ones, both in uninfected groups and in groups challenged with $M$. anisopliae. However, an experimental increase of group diversity by mixing workers originating from monogynous colonies strongly increased the survival of workers challenged with $M$. anisopliae, whereas it tended to decrease their survival in absence of infection. This experiment suggests that group diversity, be it genetic or environmental, improves the mean resistance of group members to the fungal infection, probably through the sharing of physiological or behavioural defences.
\end{abstract}

\section{Keywords}

Formica selysi, genetic diversity, Metarbizium anisopliae, parasite resistance, queen number, social evolution, social immunity, social insects.

Ecology Letters (2008) 11: 682-689

\section{INTRODUCTION}

An intriguing feature of animal societies is that they vary greatly in the number of breeders per group. In most species of social Hymenoptera (ants, wasps and bees), queens mate with a single male, but polyandry is prominent in a few taxa (Strassmann 2001). In contrast to the relative rarity and scattered taxonomic distribution of polyandry, polygyny (the stable coexistence of multiple queens reproducing in the same colony) is very common and widespread, particularly in ants (Bourke \& Franks 1995; Crozier \& Pamilo 1996).

The evolution of polyandry and polygyny has attracted much attention. Multiple mating is likely to bear some costs to the queens, and the presence of multiple queens decreases the reproductive output of each queen (Bourke \& Franks 1995; Crozier \& Fjerdingstad 2001). More importantly, both polyandry and polygyny decrease the relatedness among nestmates, which reduces the inclusive fitness of workers and the strength of selection for adaptive worker behaviour (Hamilton 1964, 1987).
Conversely, polyandry and polygyny increase the genetic diversity within the colony, which may provide various kinds of benefits. In particular, it has been proposed that higher colony genetic diversity may improve disease resistance (the 'genetic diversity for disease resistance' hypothesis, Hamilton 1987; Sherman et al. 1988), permit to tolerate a broader range of environmental conditions (Crozier \& Page 1985), or increase colony efficiency and homeostasis (Robinson 1992; Oldroyd \& Fewell 2007).

Recent models suggest that the relationship between colony genetic diversity and disease resistance is complex, because genetically heterogenous colonies are likely to be susceptible to a larger range of parasites (van Baalen \& Beekman 2006). However, if colonies are exposed to infection independently of genetic diversity, genetically heterogeneous colonies can better resist parasite under certain conditions (Brown \& Schmid-Hempel 2003; van Baalen \& Beekman 2006). Higher colony genetic diversity also tends to decrease the variance among colonies in the proportion of surviving workers, which might be beneficial 
if the relationship between the number of workers and colony fitness is convex (Sherman et al. 1988; Brown \& Schmid-Hempel 2003). More broadly, colony diversity, be it due to genetic or environmental variation, may improve the mean survival of all colony members in multiple ways. The simplest mechanism is that the presence of a given proportion of resistant individuals may confer 'herd immunity' by preventing the maintenance and spread of disease within the colony (Anderson \& May 1985). Furthermore, better disease resistance in groups may result from the transfer of resistance among colony members (Traniello et al. 2002), for example, by sharing endogenously produced antibiotics (Rosengaus et al. 2000), or when the behaviour of some specialists protects other individuals (Boomsma et al. 2005).

Several other hypotheses have been proposed to explain the evolution of polyandry, including convenience mating, bet-hedging to avoid genetic incompatibility, increased sperm store or reduced sex allocation conflict (reviewed in Crozier \& Fjerdingstad 2001). Similarly, multiple factors are likely to promote the evolution of polygyny, from ecological constraints limiting independent breeding to benefits provided by changes in dispersal, mode of colony founding and life-history traits that are associated with the presence of multiple queens (Bourke \& Franks 1995; Rosset \& Chapuisat 2007). Given the complexity and multiplicity of processes that are potentially involved in the evolution of either polyandry or polygyny, experimental data testing specific hypotheses are needed.

The 'genetic diversity for disease resistance' hypothesis proposes that social groups with higher genetic diversity better resist parasites (Hamilton 1987; Sherman et al. 1988; Shykoff \& Schmid-Hempel 1991). This hypothesis has received strong empirical support with respect to variation in mate number in monogynous species of eusocial Hymenoptera. Experiments in fungus-growing ants Acromyrmex echinatior (Hughes \& Boomsma 2004), honeybees Apis mellifera (Palmer \& Oldroyd 2003; Tarpy 2003; Tarpy \& Seeley 2006; Seeley \& Tarpy 2007) and bumblebees Bombus terrestris (Liersch \& Schmid-Hempel 1998; Baer \& SchmidHempel 1999, 2001) have shown genetic variation for resistance among patrilines and reduced disease prevalence in colonies with higher genetic diversity, at least under some conditions. This large amount of data suggest that parasites play a role in promoting the evolution of multiple-mating by the queen, even if other factors are also likely to be important (Kraus \& Page 1998; Crozier \& Fjerdingstad 2001).

The relationship between queen number and parasite resistance has received less attention, but two studies suggest that polygynous colonies might better resist parasites. A comparative analysis revealed that the level of relatedness within colonies was positively correlated with parasite loads (Schmid-Hempel \& Crozier 1999). Moreover, a brood-transfer experiment in the normally monogynous species Bombus terrestris showed that colonies with greater heterogeneity had lower parasite loads (Liersch \& SchmidHempel 1998). Hence, the effect of polygyny and group diversity on disease resistance deserves further experimental investigation in species where queen number varies. It is also important to note that, in the context of polygyny, group diversity is a broader concept than genetic diversity alone, as it also includes phenotypic variance among matrilines coming from maternal effects or variation in ecology, ontogeny and demography.

Here, we examine if group diversity improves disease resistance in experimental groups of the ant Formica selysi. This ant shows natural variation in the number of queens per colony. Monogynous and polygynous colonies coexist in close proximity in the same habitat (Chapuisat et al. 2004). In the field, polygynous colonies are genetically more diverse than monogynous ones (Rosset et al. 2007), but they are also more populous (Rosset \& Chapuisat 2007) and have slightly smaller workers that are less variable in size (Schwander et al. 2005).

We infected experimental groups with a strain of Metarizhium anisopliae var. anisopliae that we isolated from soil at our study site. This virulent fungal entomopathogen of many insect species is commonly used as an experimental pathogen of ants and termites (e.g. Hughes \& Boomsma 2004; Calleri et al. 2006; Chapuisat et al. 2007). Although the prevalence of Metarbizium appears to be low in natural populations of ants (Hughes et al. 2004), we recently found infected workers in one colony of our study population.

We evaluated the "genetic diversity for disease resistance" hypothesis, and more generally the effect of group diversity, in two ways. First, we compared the resistance of groups of workers originating from monogynous and polygynous field colonies, both in controls and when exposed to M. anisopliae. Second, we tested if increased group diversity obtained by experimentally mixing workers originating from monogynous colonies improved disease resistance.

\section{METHODS}

\section{Sampling of ants}

The ants came from a population of Formica selysi located between Sierre and Susten along the river Rhône in central Valais, Switzerland. We sampled ants from 42 monogynous and 28 polygynous field colonies on which we already had detailed genetic information (see below). Workers were collected between July and September 2005 under flat stones placed on each field colony. Measures of the head width of workers from 32 of the colonies used in the present study confirmed that workers from polygynous 
colonies are significantly smaller than workers from monogynous ones (hierarchical ANOVA with colony nested in social structure, $F_{1,21}=30.99, \quad P=0.02$; see also Schwander et al. 2005).

\section{Genetic analyses}

We checked the social structure of the field colonies by sampling and genotyping five to eight workers from each colony used in the present study at nine microsatellite markers, following the methods described in Chapuisat $e t$ al. (2004). We inspected the arrays of genotypes from the individuals sampled in our study and compared them with those of eight to 100 individuals sampled from the same field colonies in the previous years (Chapuisat et al. 2004; Schwander et al. 2005; Rosset \& Chapuisat 2006). This procedure revealed that the social structure of the sampled colonies had remained unchanged, and permitted to determine with high confidence which of the field colonies were headed by one queen, and which had multiple queens (see Chapuisat et al. 2004 for details).

We determined the average relatedness among nestmates, effective queen number and allelic richness from the genotypes of the workers sampled in our study. The relatedness among nestmate workers was computed with the computer program Relatedness 5.0.4 (http://www. gsoftnet.us/GSoft.html). Nests were weighted equally and standard errors were obtained by jack-knifing over nests. The average relatedness $(r$ ) was $0.73 \pm 0.02$ (mean $\pm \mathrm{SE}$, $95 \%$ confidence interval $0.69-0.77$ ) in monogynous colonies and $0.18 \pm 0.03(95 \%$ confidence interval $0.11-0.25)$ in polygynous colonies.

We estimated the effective number of queens from the relatedness among workers $(r)$ with the formula $(3-r) /(3 r)$, which assumes that the relatedness among queens is equal to the relatedness among workers, as expected if queens stay in their natal nest (Chapuisat $e t$ al. 2004). The effective number of queens was $1.11 \pm 0.06$ (mean $\pm \mathrm{SE}$ ) in monogynous colonies and $13.06 \pm 3.08$ in polygynous ones.

We computed allelic richness with the program FSTAT v2.9.1 (http://www2.unil.ch/popgen/softwares/fstat.htm). The average allelic richness was significantly higher in polygynous colonies than in monogynous ones (2.35 vs. 1.82 , respectively; Wilcoxon rank-sum test, $Z=6.68$, $P<0.0001)$.

\section{Parasite preparation}

We isolated a strain of Metarbizium anisopliae var. anisopliae from soil collected at our study site. Soil samples were diluted in sterile $0.8 \% \mathrm{NaCl}$ solution and plated on semiselective medium for Metarbizium (Keller et al. 2003). The Metarbizium culture was started from a single colony-forming unit presenting the characteristic green colour and stretched conidia shape, which was transferred to a nutritive medium (Malt Extract Agar). To confirm the species identification, a 543-bp long DNA fragment in the nuclear ITS1-5.8S-ITS2 region of the rRNA gene-complex was amplified by PCR with the primers TW81/AB28 and sequenced (Curran et al. 1994). The strain that we isolated perfectly matched the sequence of $M$. anisopliae var. anisopliae published in GenBank (accession number AY635457.1, found after a search with BLAST 1.4 10MP, http://www.ncbi.nlm.nih.gov/).

We cultured the strain on the nutritive medium at $25{ }^{\circ} \mathrm{C}$ for 5-7 days to obtain conidia (asexual spores). The conidia were harvested into sterile $0.05 \%$ Tween 20. Conidia concentration was quantified using a haemocytometer and adjusted to $10^{7}$ conidia $\mathrm{mL}^{-1}$. This conidia solution was used to infect ants in experimental groups.

\section{Tests of disease resistance}

In the two experiments described below, we established experimental groups of 40 individuals in plastic boxes $(13.5 \mathrm{~cm}$ long $\times 15 \mathrm{~cm}$ wide $\times 5 \mathrm{~cm}$ high) lined with fluon to prevent escape. Experimental groups were maintained in the laboratory at $25{ }^{\circ} \mathrm{C}$ under a $12 \mathrm{~h} \mathrm{day} /$ night cycle, and provided ad libitum with water and a protein-rich jelly made of honey, egg and agar.

In the first experiment, we compared the resistance of workers originating from monogynous and polygynous field colonies. We sampled workers from 27 monogynous and 28 polygynous colonies. For each field colony, we set up two experimental groups, of which one was infected with M. anisopliae and the other served as control (see below).

In the second experiment, we tested the effect of group diversity on resistance. We created three levels of diversity by briefly cooling down workers originating from monogynous field colonies, before to mix them in experimental groups. This manipulation increased within-group genetic diversity, and possibly also variation due to maternal or environmental effects. Workers of this species show little aggression in the field (Rosset et al. 2007), and they behaved peacefully in our mixed laboratory groups. Low diversity groups had 40 individuals from a single colony, medium diversity groups had 20 individuals from each of two colonies, and high diversity groups had 10 individuals from each of four colonies. We collected workers from 32 monogynous field colonies, from which we created 64, 32 and 16 experimental groups with low, medium and high diversity, respectively. For each combination of colonies, one of the two experimental groups was infected with M. anisopliae and the other served as control.

We monitored the mortality in infected and control groups. In experimental groups infected with M. anisopliae, $400 \mu \mathrm{L}$ of conidia solution were deposited daily on a filter 
paper ( $7 \mathrm{~cm}$ diameter) that was left unchanged in the middle of the plastic box, so that workers were in contact with a concentration of conidia sufficient to cause significant mortality during the entire time course of the experiment (Chapuisat et al. 2007). Control groups received $400 \mu \mathrm{L}$ of sterile $0.05 \%$ Tween 20 . The experiment lasted for 55 and 30 days in the first and second experiment, respectively.

To check the efficiency of the infection with $M$. anisopliae, all corpses were removed, surface sterilized, placed in tubes with wet cotton wool, and kept at $25{ }^{\circ} \mathrm{C}$ to check for the presence of conidia (Lacey 1997). We observed that $51.4 \%$ of the 2172 corpses from infected groups and none of the 653 corpses from control groups produced Metarhizium conidia.

\section{Statistical analyses}

We analysed ant survival with repeated-measures ANOVAs. We used mixed models in which the dependent variable (repeated measure) was the arcsin square root transformed proportion of ants alive recorded daily. The models were constructed with the function Ime (linear mixed-effects models) of the statistical package $R$ (http://www.r-project.org). We computed the ANOVA table of the model to obtain degrees of freedom, $F$-statistics and $P$-values for the fixed factors.

To compare the survival of workers originating from monogynous and polygynous field colonies, the social structure of origin (monogynous or polygynous) and infection (control or challenged with $M$. anisopliae) were fixed factors, and experimental colony (each box containing a group of workers) was a random factor. Such procedure was chosen because the use of the proportion of surviving ants controls for the non-independence of workers in the same box at any point in time, whereas the random factor 'experimental box' controls for the non-independence of the repeated measures, as each box may be affected by some uncontrolled factor influencing the survival of ants over time. A significant interaction between social structure, infection and day of measure is expected if workers originating from monogynous and polygynous field colonies differ in their response to infection over time. We further investigated the differences in survival between workers from monogynous and polygynous colonies with repeatedmeasures ANOVA within the uninfected or infected treatments, respectively.

The effect of the experimental manipulation of group diversity on workers' resistance was analysed with a similar model. Group diversity (low, medium and high) and infection (control or challenged with $M$. anisopliae) were fixed factors, and experimental colony (each box containing a group of workers) was a random factor. A significant interaction between group diversity, infection and day of measure is expected if groups with different diversity levels vary in their response to infection over time. To further investigate the direction of this effect, we also applied repeated-measures ANOVA between levels of group diversity within the uninfected or infected treatments, respectively.

\section{RESULTS}

Workers originating from monogynous and polygynous field colonies did not differ significantly in their response to an experimental infection with $M$. anisopliae, as shown by the absence of interaction between social structure, infection and day of measure (Fig. $1, F_{1,4669}=0.27, P=0.6$ ). The infection with $M$. anisopliae strongly reduced the survival of workers originating from both types of colonies (Fig. 1, interaction between infection and day of measure, $\left.F_{1,4669}=553.5, P<0.0001\right)$. Interestingly, workers originating from monogynous and polygynous colonies differed significantly in survival, independently of infection (Fig. 1, interaction between social structure and day of measure, $\left.F_{1,4669}=64.9, P<0.0001\right)$. Specifically, workers originat-
Figure 1 Resistance of workers from monogynous and polygynous field colonies. Survival (mean \pm SE) of workers originating from monogynous (circles) or polygynous (squares) colonies in uninfected control groups (open symbols) or groups infected with $M$. anisopliae (filled symbols).

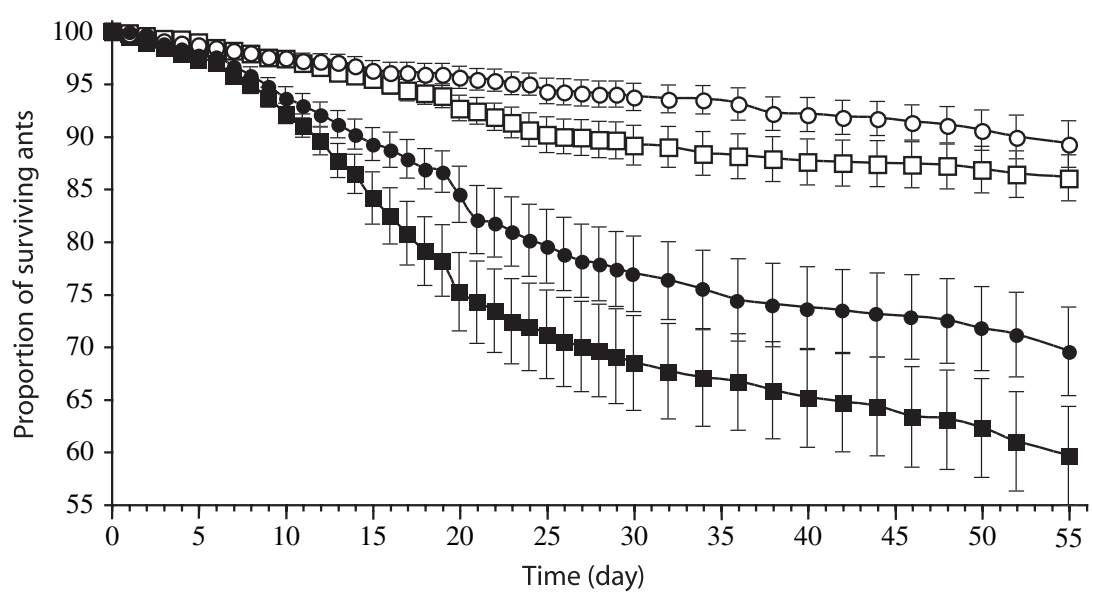

(C) 2008 Blackwell Publishing Ltd/CNRS 


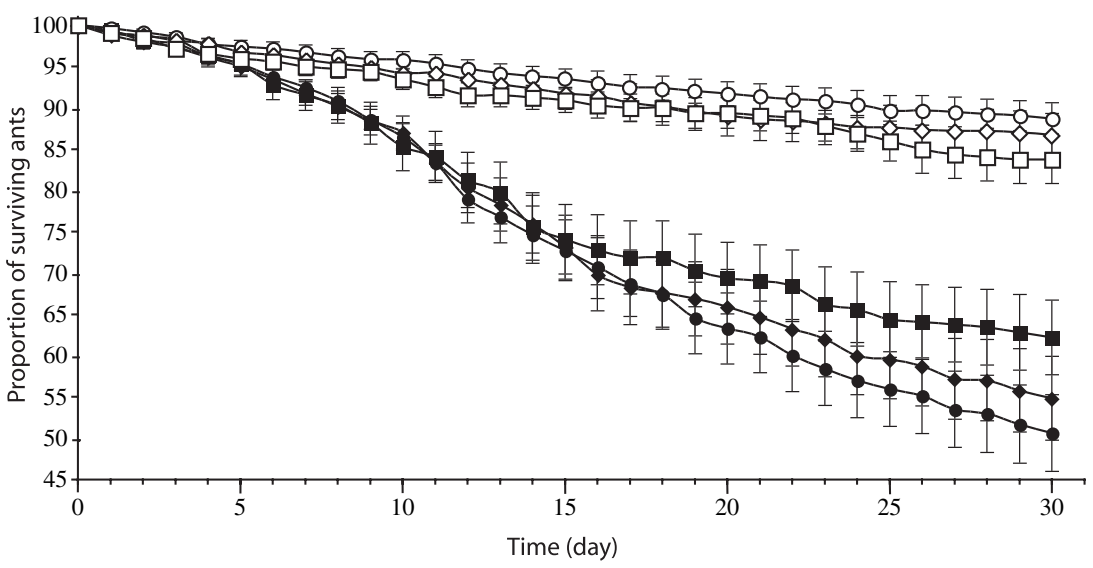

Figure 2 Impact of group diversity on disease resistance. Survival (mean $\pm \mathrm{SE}$ ) of workers in low (circles), medium (diamonds) or high (squares) diversity groups that were uninfected (open symbols) or infected with M. anisopliae (filled symbols).

ing from monogynous colonies had higher survival than workers originating from polygynous colonies, both within uninfected control groups (Fig. 1, $\quad F_{1,2308}=80.1$, $P<0.0001)$ and within groups infected with $M$. anisopliae (Fig. 1, $F_{1,2308}=37.8, P<0.0001$ ).

The experimental manipulation of diversity in groups of workers originating from monogynous colonies had a strong impact on the resistance to the fungal infection, as shown by the significant interaction between group diversity, infection and day of measure (Fig. 2, $F_{2,3354}=21.5, P<0.0001$ ). When challenged with $M$. anisopliae, workers had significantly higher survival in high diversity groups originating from four colonies than in medium diversity groups originating from two colonies $\left(F_{1,718}=8.0, P<0.01\right)$ or low diversity groups originating from a single colony $\left(F_{1,1198}=29.2, P<0.0001\right)$. Similarly, workers challenged with $M$. anisopliae survived better in medium diversity groups than in low diversity groups $\left(F_{1,1438}=9.9, P<0.01\right)$. In contrast, in absence of infection, workers in low diversity groups survived significantly better than those in high diversity groups $\left(F_{1,1198}=4.7, P<0.05\right)$, whereas the survival of workers did not differ significantly between high and medium diversity groups $\left(F_{1,718}=1.6, P=0.20\right)$, nor between medium and low diversity groups $\left(F_{1,1438}=\right.$ $0.63, P=0.42)$.

\section{DISCUSSION}

F. selysi workers originating from monogynous field colonies had higher survival than workers originating from polygynous colonies, and this both in uninfected groups and in groups challenged with the fungal parasite $M$. anisopliae. Hence, natural variation in group genetic diversity in the field did not correlate with improved parasite resistance, which provides no support to the 'genetic diversity for disease resistance' hypothesis in this context. In certain conditions, lower group diversity might even be associated with reduced disease susceptibility (Lewis 1998; Thorne \& Traniello 2003).
For example, in the termite Zootermopsis angusticollis primary reproductives suffered less from parasite-induced mortality when they paired with nestmates than non-nestmates (Rosengaus \& Traniello 1993; Calleri et al. 2005).

The effect of natural variation in genetic diversity among colonies in our field population is however difficult to assess, because previous studies have revealed that the shift from monogynous to polygynous colonies is associated with multiple changes not only in genetic diversity, but also in colony size, colony longevity, worker body size, and investment in reproduction and maintenance (Chapuisat et al. 2004; Schwander et al. 2005; Rosset \& Chapuisat 2007). In particular, polygynous colonies have approximately 10 times more workers than monogynous ones (Rosset \& Chapuisat 2007), and these workers are significantly smaller and less variable in size (Schwander et al. 2005). These pronounced differences in resources allocation might explain why workers originating from monogynous colonies had higher survival than workers originating from polygynous ones, both in controls and when infected with $M$. anisopliae. Further experiments will be necessary to disentangle the effects of worker size and other correlates of queen number variation on disease resistance, including indirect effects of colony size and demography (Rosengaus et al. 1998; Rosengaus \& Traniello 2001).

As multiple factors covary with queen number in the field, a proper test of the effect of group diversity on disease resistance requires to experimentally manipulate diversity among groups of workers originating from the same type of colonies. Indeed, the experimental increase of group diversity by mixing workers originating from monogynous field colonies strongly increased the mean survival of workers challenged with $M$. anisopliae. This pattern in presence of the parasite contrasts sharply with a tendency for lower survival in more diverse groups in absence of infection. These results suggest that, other things being equal, group diversity improves the mean resistance of group members to the fungal infection. 
The design of the experiment was such that all workers were repeatedly exposed to the pathogen. Moreover, the cadavers were removed before they could release new conidia, so that there was no disease transmission from dead to live individuals. Hence, in our experiment group diversity was unlikely to improve mean survival by reducing the number of susceptible individuals below some threshold preventing the spread of epidemics (herd immunity, Anderson \& May 1985), or by limiting parasite adaptation in more genetically diverse hosts (e.g. Hughes \& Boomsma 2006).

Given that the fungus was not transmitted from dead to live ant, the higher survival in more diverse groups suggests that group diversity increased the mean defences of workers towards the fungal pathogen, probably through a transfer of physiological defences or a sharing of behavioural defences. One possibility is that workers share endogenously produced antifungal substances that vary among lineages. For example, they might exchange substances secreted by their metapleural glands (Fernandez-Marin et al. 2006). Workers from some lineages might also be particularly efficient at removing or neutralizing $M$. anisopliae conidia, thus conferring protection to other individuals (Jaccoud et al. 1999; Hughes et al. 2002).

The positive effect of group diversity on worker's resistance is in line with the finding that multiple mating in Acromyrmex ants or outbreeding in Zootermopsis termites mitigate against the effect of $M$. anisopliae, at least for some doses of the parasite (Hughes \& Boomsma 2004; Calleri et al. 2006). It is also consistent with experiments showing that genetic diversity reduces parasite load in bumblebee colonies (Liersch \& Schmid-Hempel 1998; Baer \& SchmidHempel 1999, 2001), and diminishes the impact of bacterial or fungal pathogens in honeybee colonies (Tarpy 2003; Tarpy \& Seeley 2006; Seeley \& Tarpy 2007).

In our experiment, the positive effect of group diversity was only observed in groups infected with $M$. anisopliae. In contrast, in absence of infection workers in low diversity groups survived better than those in high diversity groups. This result suggests that the mixing of workers from different colonies had a negative impact on the ants. Although $F$. selysi workers recognize non-nesmates in the field, they are not very aggressive (Rosset et al. 2007), and we did not observe any aggression or segregation in our queenless laboratory groups. However, it is possible that stress or cryptic antagonistic interactions caused higher mortality in more diverse group in absence of infection. In any case, the positive effects of group diversity against $M$. anisopliae had to be strong to reverse the trend observed in uninfected groups.

It should also be noted that part of the group diversity in our experiment may not be genetic. Indeed, the phenotypic differences among workers sampled in different field colonies are likely to be partly due to maternal or environmental effects. Importantly, maternal effects are also expected to contribute to phenotypic differences among matrilines within colonies. This alternative source of variation might be of lower magnitude than genetic effects, but is also relevant for the hypothesis that greater queen number improves disease resistance.

In conclusion, the better resistance of workers in groups with experimentally increased diversity suggests that, other things being equal, parasites have less impact in colonies with multiple matrilines. It is consistent with the "genetic diversity for diseases resistance hypothesis" and with the idea that the pressure of parasites is one of the factors favouring the evolution of polygyny (Hamilton 1987). Part of the positive effect of group diversity may also stem from between-lineage variation in maternal effects, ontogeny, demography and ecology. Hence, further experiments with lab-reared workers will be needed to distinguish between the various sources of group diversity.

We did not detect a positive effect of genetic diversity when comparing the disease resistance of workers originating from monogynous and polygynous field colonies, in which the genetic diversity had not been manipulated but vary naturally. The most likely explanation for this pattern is that some of the many factors that covary with queen number variation in the field have antagonistic effects on parasite resistance. Polygynous field colonies have a much larger number of workers than monogynous ones (Rosset \& Chapuisat 2007) and these workers have a significantly smaller body size (Schwander et al. 2005), which might explain why they did not show increased resistance to $M$. anisopliae despite the higher genetic diversity in their groups. Together, these results exemplify the importance of experimentally manipulating single factors rather than relying on correlational field data when studying complex evolutionary processes.

\section{ACKNOWLEDGEMENTS}

We thank Hervé Rosset, Nathalie Tzaud and Christophe Hug for help in the field and in the laboratory, Julien Gasparini for advice on statistics, and Rob Hammond, Laurent Keller, Gabriele Sorci, James Traniello and two anonymous referees for comments on the manuscript. This research was supported by grants 3100A0-104118 and 3100A0-108263 from the Swiss National Science Foundation.

\section{REFERENCES}

Anderson, R.M. \& May, R.M. (1985). Vaccination and herd immunity to infectious diseases. Nature, 318, 323-329.

van Baalen, M. \& Beekman, M. (2006). The costs and benefits of genetic heterogeneity in resistance against parasites in social insects. Am. Nat., 167, 568-577. 
Baer, B. \& Schmid-Hempel, P. (1999). Experimental variation in polyandry affects parasite loads and fitness in a bumble-bee. Nature, 397, 151-154.

Baer, B. \& Schmid-Hempel, P. (2001). Unexpected consequences of polyandry for parasitism and fitness in the bumblebee, Bombus terrestris. Evolution, 55, 1639-1643.

Boomsma, J.J., Schmid-Hempel, P. \& Hughes, W.O.H. (2005). Life histories and parasite pressure across the major groups of social insects. In: Insect Evolutionary Ecology (eds Fellowes, M.D.E., Holloway, G.J. \& Rolff, J.). CABI Publishing, Wallingford, pp. 139-175.

Bourke, A.F.G. \& Franks, N.R. (1995). Social Evolution in Ants. Princeton University Press, Princeton, NJ.

Brown, M.J.F. \& Schmid-Hempel, P. (2003). The evolution of female multiple mating in social hymenoptera. Evolution, 57, 2067-2081.

Calleri, D.V., Rosengaus, R.B. \& Traniello, J.F.A. (2005). Disease and colony foundation in the dampwood termite Zootermopsis angusticollis: the survival advantage of nestmate pairs. Naturwissenschaften, 92, 300-304.

Calleri, D.V., Reid, E.M., Rosengaus, R.B., Vargo, E.L. \& Traniello, J.F.A. (2006). Inbreeding and disease resistance in a social insect: effects of heterozygosity on immunocompetence in the termite Zootermopsis angusticollis. Proc. R Soc. Lond. B, 273, 2633-2640.

Chapuisat, M., Bocherens, S. \& Rosset, H. (2004). Variable queen number in ant colonies: no impact on queen turnover, inbreeding, and population genetic differentiation in the ant Formica selysi. Evolution, 58, 1064-1072.

Chapuisat, M., Oppliger, A., Magliano, P. \& Christe, P. (2007). Wood ants use resin to protect themselves against pathogens. Proc. R Soc. Lond. B, 274, 2013-2017.

Crozier, R.H. \& Fjerdingstad, E.J. (2001). Polyandry in social Hymenoptera - disunity in diversity? Ann. Zool. Fenn., 38, $267-$ 285.

Crozier, R.H. \& Page, R.E. (1985). On being the right size: male contributions and multiple mating in social Hymenoptera. Behav. Ecol. Sociobiol., 18, 105-115.

Crozier, R.H. \& Pamilo, P. (1996). Evolution of Social Insect Colonies: Sex Allocation and kin Selection. Oxford University Press, Oxford.

Curran, J., Driver, F., Ballard, J.W.O. \& Milner, R.J. (1994). Phylogeny of Metarbizium: analysis of ribosomal DNA-sequence data. Mycol. Res., 98, 547-552.

Fernandez-Marin, H., Zimmerman, J.K., Rehner, S.A. \& Wcislo, W.T. (2006). Active use of the metapleural glands by ants in controlling fungal infection. Proc. $R$ Soc. Lond. B, 273, 1689-1695.

Hamilton, W.D. (1964). The genetical evolution of social behaviour. II. J. Theor. Biol., 7, 17-52.

Hamilton, W.D. (1987). Kinship, recognition, disease, and intelligence: constraints of social evolution. In: Animal Societies: Theories and Facts (eds Ito, Y., Brown, J.L. \& Kikkawa, J.). Japan Sci. Soc. Press, Tokyo, pp. 81-102.

Hughes, W.O.H. \& Boomsma, J.J. (2004). Genetic diversity and disease resistance in leaf-cutting ant societies. Evolution, 58, $1251-1260$

Hughes, W.O.H. \& Boomsma, J.J. (2006). Does genetic diversity hinder parasite evolution in social insect colonies? J. Evol. Biol., 19, 132-143.

Hughes, W.O.H., Eilenberg, J. \& Boomsma, J.J. (2002). Trade-offs in group living: transmission and disease resistance in leaf-cutting ants. Proc. R Soc. Lond. B, 269, 1811-1819.
Hughes, W.O.H., Thomsen, L., Eilenberg, J. \& Boomsma, J.J. (2004). Diversity of entomopathogenic fungi near leaf-cutting ant nests in a neotropical forest, with particular reference to Metarbizium anisopliae var. anisopliae. J. Invertebr. Pathol., 85, 46-53.

Jaccoud, D.B., Hughes, W.O.H. \& Jackson, C.W. (1999). The epizootiology of a Metarbizium infection in mini-nests of the leafcutting ant Atta sexdens rubropilosa. Entomol. Exp. Appl., 93, 51-61.

Keller, S., Kessler, P. \& Schweizer, C. (2003). Distribution of insect pathogenic soil fungi in Switzerland with special reference to Beauveria brongniartii and Metarbizium anisopliae. Biocontrol, 48, 307319.

Kraus, B. \& Page, R.E. (1998). Parasites, pathogens, and polyandry in social insects. Am. Nat., 151, 383-391.

Lacey, L.A. (1997). Manual of Techniques in Insect Pathology. Academic Press, San Diego.

Lewis, K. (1998). Pathogen resistance as the origin of kin altruism. J. Theor. Biol., 193, 359-363.

Liersch, S. \& Schmid-Hempel, P. (1998). Genetic variation within social insect colonies reduces parasite load. Proc. $R$ Soc. Lond. B, 265, 221-225.

Oldroyd, B.P. \& Fewell, J.H. (2007). Genetic diversity promotes homeostasis in insect colonies. Trends Ecol. Evol., 22, 408-413.

Palmer, K.A. \& Oldroyd, B.P. (2003). Evidence for intra-colonial genetic variance in resistance to American foulbrood of honey bees (Apis mellifera): further support for the parasite/pathogen hypothesis for the evolution of polyandry. Naturwissenschaften, 90 , 265-268.

Robinson, G.E. (1992). Regulation of division of labor in insect societies. Annu. Rev. Entomol., 37, 637-665.

Rosengaus, R.B. \& Traniello, J.F.A. (1993). Disease risk as a cost of outbreeding in the termite Zootermopsis angusticollis. Proc. Natl Acad. Sci. USA, 90, 6641-6645.

Rosengaus, R.B. \& Traniello, J.F.A. (2001). Disease susceptibility and the adaptive nature of colony demography in the dampwood termite Zootermopsis angusticollis. Behav. Ecol. Sociobiol., 50, 546-556.

Rosengaus, R.B., Maxmen, A.B., Coates, L.E. \& Traniello, J.F.A. (1998). Disease resistance: a benefit of sociality in the dampwood termite Zootermopsis angusticollis (Isoptera : Termopsidae). Behav. Ecol. Sociobiol., 44, 125-134.

Rosengaus, R.B., Lefebvre, M.L. \& Traniello, J.F.A. (2000). Inhibition of fungal spore germination by Nasutitermes: Evidence for a possible antiseptic role of soldier defensive secretions. J. Chem. Ecol., 26, 21-39.

Rosset, H. \& Chapuisat, M. (2006). Sex allocation conflict in ants: when the queen rules. Curr. Biol., 16, 328-331.

Rosset, H. \& Chapuisat, M. (2007). Alternative life-histories in a socially polymorphic ant. Evol. Ecol., 21, 577-588.

Rosset, H., Schwander, T. \& Chapuisat, M. (2007). Nestmate recognition and levels of aggression are not altered by changes in genetic diversity in a socially polymorphic ant. Anim. Behav., 74, 951-956.

Schmid-Hempel, P. \& Crozier, R.H. (1999). Polyandry versus polygyny versus parasites. Philos. Trans. $R$ Soc. Lond. B, 354, 507-515.

Schwander, T., Rosset, H. \& Chapuisat, M. (2005). Division of labour and worker size polymorphism in ant colonies: the impact of social and genetic factors. Behav. Ecol. Sociobiol., 59, 215221.

Seeley, T.D. \& Tarpy, D.R. (2007). Queen promiscuity lowers disease within honeybee colonies. Proc. $R$ Soc. Lond. B, 274, 6772 . 
Sherman, P.W., Seeley, T.D. \& Reeve, H.K. (1988). Parasites, pathogens, and polyandry in social Hymenoptera. Am. Nat., 131, 602-610.

Shykoff, J.A. \& Schmid-Hempel, P. (1991). Parasites and the advantage of genetic variability within social insect colonies. Proc. $R$ Soc. Lond. B, 243, 55-58.

Strassmann, J. (2001). The rarity of multiple mating by females in the social Hymenoptera. Ins. Soc., 48, 1-13.

Tarpy, D.R. (2003). Genetic diversity within honeybee colonies prevents severe infections and promotes colony growth. Proc. $R$ Soc. Lond. B, 270, 99-103.

Tarpy, D.R. \& Seeley, T.D. (2006). Lower disease infections in honeybee (Apis mellifera) colonies headed by polyandrous vs monandrous queens. Naturwissenschaften, 93, 195-199.
Thorne, B.L. \& Traniello, J.F.A. (2003). Comparative social biology of basal taxa of ants and termites. Annu. Rev. Entomol., 48, 283-306. Traniello, J.F.A., Rosengaus, R.B. \& Savoie, K. (2002). The development of immunity in a social insect: evidence for the group facilitation of disease resistance. Proc. Natl Acad. Sci. USA, 99, 6838-6842.

Editor, Gabriele Sorci

Manuscript received 14 November 2007

First decision made 13 December 2007

Second decision made 15 February 2008

Manuscript accepted 26 February 2008 